immer sofort in Ulceration übergebt, so z. B. in der Hornhaut, den Knochen, Knorpeln, vielleicht auch in den sehr festen Formen des Bindegewebes (Selinen). Ich finde den Grund davon in dem Umstande, dass die starre Beschaffenheit dieser Gewebe den neugebildeten Zellen den Austritt verwehrt und die Ansammlung derselben daher nothwendig zum Zerfall der Grundsubstanz führen muss.

Königsherg, im December 1861.

8.

\title{
Ueber Eiterbildung.
}

Von Rud. Virchow.

Die vorstehenden Bemerkingen des Hrn. E. Neumann nöthigen mich, einige Angaben zur Berichtigung der mir darin zugeschriebenen Behauptungen zu machen. Der Verfasser erklärt zunächst, dass er, gegenüber der von mir u. A. angenommenen epithelialen Abstammung der Eiterzellen bei Entzündung der serösen und Schleimhäute, die Ansicht ausgesprochen habe, die Eiterzellen gingen hauptsächlich oder vielleicht ausschliesslich aus Proliferation des Bindegewebes dieser Membranen hervor. Sodann betont er vor Allem, dass bei der Eiterbildung von serösen Häuten keine Ejnschmelzung der Intercellularsubstanz stattfinde, der Eiter also kein geschmolzenes, transformirtes Gewebe sei, wie ich angegeben habe, sondern dass die Eiterkörperchen durch das Exsudat aus der unversehrt bleibenden Intercellularsubstanz hervorgespült würden.

In ersterer Beziehung muss ich zunächst darauf aufmerksam machen, dass ich wesentlich unterschieden habe and unterscheide zwischen einer oberfläch lichen (epithelialen) und einer tiefen (Bindegewebs-) Eiterung. Man vergleiche die Cellularpathologie 1ste Aufl. S. 396 u. 400, 3te Aufl. S. 415 u. 419. Diess gilt am meisten für die äussere Haut, die Schleim- und serösen Häute, und wenn mir nachgeredet werden sollte, dass ich jemals für diese Theile die tiefe Eiterung geleugnet habe, so glaube ich wenigstens dazn keine Veranlassung gegeben zu haben. Vielmehr habe ich lange vor der Zeit, wo Andere daran dachten, solehe Fragen aufzuwerfen, gerade diese tiefe Eiterung nachgewiesen und beschriehen. Die betreffende Stelle, auf welche ich übrigens schon in meinem listorischen Rückblick auf die neuere Entwicklung der Eiterlehre (Archiv XV. S. 531) besonders hingewiesen habe, findet sich in meiner ersten Arbeit über die parenchymatöse Entzündung (Arcbiv 1852. IV. S. 312), und wenn ich sie wörtlich citire, so geschieht es, weil daraus zugleich ersichtlich wird, dass auch die Erkenntniss keine neue ist, dass "ein mit Eiterzellen infiltrirtes Bindegewebe sich ohne Verlust von Intercellularsubstanz der Zellen entledigen könne". Ich sagte damals; „Es bilden sich hier (im Bindegewebe) ähnliche Heerde endogen wuchernder Elemente, wie bei den Knorpeldegenerationen, und in ähnlicher Weis e brechen diese Heerde an 


\section{6}

der Oberfäche auf, ergiessen ihren Inhalt nach aussen und bedingen so eine sebr eigenthümliche Form der Ulceration, wie sie an der äusseren Haut und den Synovialhäuten nicht so selten zu seken ist. Mauches, was man als Eiter in dem Sinne cines reinen Exsudates zu diagnosticiren plegt, stammt von der Entleerung dieser Heerde, und nicht alle "Absonderung" auf der Geschwürsfläche stammt aus den Gefässen, manche ist wirklich auf das Gewebe zurückzufüliren."

An diese Mittheilung schloss sich sehr bald eine weitere Beobachtungsreihe über den Katarrh des Kehlkopfes an, welche einer der sorgfältigsten meiner Schüler, Hr. Rheiner, veröfentlichte (Archiv 1853. V. S. 560). Darin wurde die Betbeiligung des Bindegewebes an der katarrhalischen Erkrankung und die daraus hervorgehende Ulceration genauer dargelegt. Es ist das freilich etwas lange her und jetzt, nach 10 Jahren, kann immerhin ein neues Geschlecht von Untersuchern, unbewusst, auf wessen Schultern stehend es fortarbeitet, dasselbe von Neuem entdecken. Nur berechtigt das nicht, den alten Beobachtern "Annahmen" unterzuschieben, wo sie gewissenhaft beobachtet haben, oder die eine Seite ibrer Beobachtungen für unrichtig za erhlären, weil man zufälligerweise nur die andere verfolgt hat. Hr. Neumann musste überdiess wissen, dass mir die Zeit fehlt, um alle Detailbeobachtungen, die ich mache, zu veröffentlichen, und dass, wenn ich in einer summarischen Zusammenfassung, wie in den Vorlesungen über Cellularpathologie, Angaben mache, ohne sie durch Detailbeobachtungen zu stützen, daraus noch nicht folgt, dass es "Annahmen“ waren.

Für die Sclleimlaut der Harnwege haben G. Burckhardt und O. Beckmann (Archiv 1859. XVII. S. 114, 121) die tiefe oder Bindegewebs-Eiterung genauer beschrieben, indem sie auf dem Wege fortschritten, welcher durch jene älteren Untersnchungen angebahnt war. Hr. E. Neumann und nach ihm Hr. Cohnheim haben das Verdienst, für gewisse, serōse Hänte dieselbe Richtung eingehalten und eine grössere`Zahl beglaubigter Erfahrungen gesammelt zu haben. Nur so wird wahrscheinlich eine Haut nach der anderen, ein Ort nach dem anderen an die Reihe kommen und jede gute Untersuchung wird die Zuverlässigkeit unseres Wissens stärken. Insbesondere für diejenigen, welche nicht selbst untersuchen, wird auf diese Weise die Deberzeugung immer sicherer festgestellt werden, dass es eine tiefe, aus den Elementen des Bindegewebes hervorgehende Eiterung gibt.

Sicherlich folgt aus dieser Erfahrung nicht das Mindeste für die Existenz einer oberfiachlichen, epithelialen Eiterung. Dass jedoch eine solche besteht, davon wird sich jeder leicbt überzeugen können, welcher pustulöse Entzündungen der äusseren Hant, Vaginal- oder Conjunctival-Catarrhe genauer verfolgt. Schwieriger ist es an den meisten anderen Scbleim- und den serösen Häuten, doch bin ich nach meinen Untersuchungen nicht zweifelhaft, dass auch hier die Eiterbildung aus Epithel vorkommt. Aber ebenso sicher ist es, dass in Beziehung auf die Häufigkeit und Ausdehnung der epithelialen Eiterung die Erfahrungen des einen Ortes oder der einen Haut in keiner Weise maassgebend sind für andere Orte oder Häute. Ich hin z. B. darin ganz einverstanden, dass die serösen Häute viel häufiger eine Bindegewebs-Eiterung erfahren, als man sich a priori vorstellen möchte, ja dass jede grössere Eiterbildung an iknen auf das Bindegewebe übergreift. Ich 
habe selbst bemerkt, dass an den verschiedenen Schleimhäuten ganz verschiedene Verhältnisse in Beziehung auf epitheliale Eiterung bestehen (Cellularpathologie 1ste Aufl. S. 397, 3te Aufl. S. 416). Ueberall kommt es also darauf an, genau zu specialisiren. Erst dann gewinnt man nicht nur sichere wissenschaftliche Grundlagen, sondern auch wichtige praktische Anbaltspunkte.

In jedem Falle handelt es sich aber bei der Eiterbildung um eine Gewebstransformation. Denn man wird doch gewiss zugestehen müssen, dass Bindegewebe, dessen Zellen in suppurative Wucherung gerathen sind, nicht mobr Bindegewebe ist, wenn auch seine Intercellularsubstanz noch vorhanden ist. Einen solchen Zustand nennen wir bekanntlich eiterige Infiltration, nach einer alten, freilich auf anderen Vorstellungen beruhenden Ueberlieferung (Cellularpathol. 3te Aufl. S. 420). Ich bin natürlich fern davon, diese "Infiltrationen" zu leugnen, die man ja alle Tage selien kann, und ich habe nie daran gedacht, ein so transformirtes Gewebe ein gesclmoizenes zu nennen. Die Frage von der Schmelzung der Gewebe stellt sich natürlich nur da, wo Eiter im gewöhnlichen Sinne des Wortes, d. h. als Flüssigkeit vorhanden ist, und zwar zunächst da, wo er an die Stelle früher fester Gewebe getreten, denselben substituirt ist. Denn diess ist ja eben der Fall, der in der Chirurgie und Medicin seit alten Zeiten so viel discutirt ist, und der bald in der Art beschrieben ist, dass der "transsudirte“ Eiter chemiseh auf die Gewebsbestandtheile, mit denen er in Berührung kommt, Jösend einwirke, bald in der Art, dass das Gewebe sich selbst löse, ,abscedire“, der Eiter also "secernirt" werde. Darauf hin hatte ich in einer meiner Vorlesungen (Cellularpath. 1ste Auf. S. 396, 3te Aufl. S. 415) die Frage gestellt: ist der Eiter das Schmelzende oder das Geschmolzene? Diese Frage beantwortete ich dahin, dass das Letztere der Fall sei und dass insbesondere Maturation des Eiters die zunehmende Erweichung, Auflösung, Schmelzung der Intercellularsubstanz bedente (vgl. Ebendas. 1ste Aufl. S. 375, 3te Aufl. S. 409). Aber es versteht sich von selbst, dass die Frage keinen Bezug haben konnte auf solche Fälle, wo überbaupt keine Erweichung, Auflösung oder Schmelzung statffindet.

Diess ist z. B. der Fall bei vielen epithelialen Fiterungen, wo gar keine Intercellularsubstanz präexistirt, welche schmelzen könnte. Hier wird, wie ich anführte (Ebendas. 1ste Auf. S. 398, 3te Aufl. S. 417), der sich bildende Eiter "entweder durch nachwachsende Eitermasse weggedrängt, oder es erfolgt gleichzeitig eine 'Transsulation von Flüssigkeit, welche die Eiterzellen von der Oberflache entfernt." Den letzteren Vorgang verglich ich mit dem bei der Samensecretion eintretenden, und ich glaubte mich dadurch so deutlich gemacht zu haben, wie irgend möglich. Wenn nun Hr. Neumann, um seinen Gegensatz gegen mich hervorzaheben, besonders betont, dass der Eiter der serösen Häute ,ein blosses Exsudat, welchem sich die Eiterzellen aus dem Bindegewebe beigesellt haben", nad nicht ein geschmolzenes, transformirtes Gewebe sei, so kann ich einen solchen Gegensatz in keiner Weise anerkennen. Denn ich stelle nirgends in Abrede, dass sich zu jeder Eiterung eine Transsudation von Flüssigkeiten aus dem Blute hinzugesellen oder umgekehrt, dass jede Art von Transsudation sich mit Eiter vermischen kann. Im Gegentheil hin ich ganz und gar der Ansicht, dass der dünne, wässrige, 
schlechte Eiter in der Regel einen sehr grossen Antheil einfach transsudativer Flüssigkeit enthält. Diess gilt nicht bloss für die epitheliale Eiterung, sondern noch viel mehr für die tiefere, ulcerative Bindegewebseiterung, und wenn ich es nicht besonders erwähnt habe, sn linde ich vielfeicht darin eine Entseluldigung, dass ich keine Monographie über Eiter schreiben wolite und die Sache übrigens sich als von selbst verstehend betrachtete.

Folgt nun aber darans, dass der Eiter der serösen Häute ein blosses Exsudat sei, dem sich Eiterzellen beigesellt haben? Gewiss nicht. Mit eben so viel Recht könnte jemand behaupten, Blot sei Trinkwasser, dem sich Blutzellen beigesellt Laben. Wie es kèn Blut obne Blutkörperchen gibt, so auch keinen Eiter ohne Eíterkőrperchen. Die Zellen sind das Wesentliche, die Flüssigkeit das Hjazukommerde. Wenn nun ein "eiteriges Exsudat" aus Eiter und Transsudat bestelit, so wird es immer darauf ankommen, ob mehr Eiter oder mehr Transsudat darin vorhanden ist. Ist melur Transsudat darin, so werden wir die Flüssiglseit nicht Eiter nennen, so wenig als wir ein hämorrhagisches Exsudat Blut nennen. Ist dagegen mehr Eiter darin, so setzt diess auch immer eine starke Gewebsmetamorphose voraus, denn das leugnet ja niemand mehr, dass Eiterlxörperchen neugebildete, aus alten Gewebselementen hervargegangene Zellen sind.

Auch hier reducirt sich demnach das Besondere in der Anscliauung des Hrn. Neumann auf eine Nebensache, darauf nämlich, dass er eine Veränderung, namentlich eine Erweichung der Intercellularsubstanz lengnet, und dass er die aus der Wucherung der Bindegewebskörperchen hervorgehenden Eiterkörperchen durch das Transsudat einfach ausspülen lässt. Dagegen bemerke ich, dass es jedenfalls irrig ist, wenn er einen solchen Vorgang bis tief in das Gewebe, selbst his in die tiefste Lage der Darm-Serosa oder gar bis in die Muscularis hinein stattfindea lässt. Hier könnte höchstens eine Verwechselung mit Lymphgefässen geschehen sein. Als allgemeine Regel muss auch für die serösen Hâute gelten, dass die Intercellularsubstanz sich verändert, dass sie fibrinös, oder mucinös, oder albuminös wird and dass bei einer vollständigen, gu ten Eitcrung sie endlich zerfliesst. Für jemand, der unter Anwendung chemischer Reagentien diese Vorgänge vertolgt, der sich experimentelle Belege gesucht hat, die so leicht zu haben sind, kann darüber nicht der mindeste Zweifel bestehen.

Hr. Neumann gesteht für die festen Gewebe, Hornhaut, Knochen, Knorpel, vielleicht Sehnen, zu, dass hier die Suppuration "in der Regel oder immer" in Ulceration übergeht. Ich selhe davon ab, dass, wenn er sich mit diesen Geweben eingehend beschäftigt hätte, er auch an der Hornhaut und den Selnen eine eiterige Infiltration kennen gelernt haben würde; es genügt $z u$ constatiren, dass er hier die Ulceration zulässt, welehe doch die Schmelzung voraussetzt. In weichent Bindegewebe aber soll nach ihm eine Abscesshöhle durch Auseinanderdrängung des Gewebes entsteben. Hier kann ich nưr den Rath geben, die Ränder irgend einer Abscessböhle, so lange sie frisch ist, genau zu untersuchen. Man wird sich dann überzeugen, dass der Verlauf der Faserzïge und Bündel des Gewebes durch den Fiter unterbrochen wird. Sind zugleich specifische Elenente vorhanden, z. B. Muskel- oder Nervenfasern, so kann man dus leicht vom blossen Auge seben; 
aber auch dann, wenn nur Bindegewebe vorhanden ist, kann man in der Regel beim Ausspülen in Wasser die frei herrorstehenden Fetzen der unterbrochenen Bündel wabrnehmen. Jedenfalss ergibt das Mikroskop die deutliche Anschaunng, und wenn man sei es die Ränder, selbst an der schon gebildeten Abscesshöhle, sei es die Entwicklung der letzteren wälrend ihrer Bildung verfolgt, so wird man dieselbe Erweichung der Grundsubstanz constatiren bönnen, welche an festen Geweben so offenbar ins Auge fällt.

Aber, fragt Hr. Neumann, warum geht denn bicht bisweilen die ganze Serosa unter der Eiterung verloren und warum sollen nur die oberflächlichsten Schichten einschmelzen, da doch die Proliferation der Bindegewebselemente bis in die Darmmuscularis reicht? Auf den ersteren Punkt lässt sich nur sagen, dass bei chronischer Eiterung der Pleura, des Peritonaeum u. s. w. in der That nicht selten die ganze Serosa einschmilzt, zerstört wird, ja dass sogar Perforationen in die Lungen, in den Darm u. s. w. vorkommen. Bei leịchteren und namentlich bei acuten Fällen findet diess freilich in der Rege! nicht statt, und zwar desshalb nicht, weil die Proliferation der tieferen Lagen nicht bis zur Supparation, sondern nur bis zur Granulation fortsclureitet, und weil in den oberflächlichen Schichten die Wucherung so reichlich ist, dass neben den sich ablösenden, eiterigen Theilen immer noch ein gewisser Grundstock zurückbleibt, der die fernere Wucherung unterhält. Gesetzt, ein ursprïnglicher Gewebstheil a theilte sich in 2 neue: $a^{\prime}$ and $b$, beide wiederum in je $z$ neue: $a^{\prime \prime}$ und $c, b^{\prime \prime}$ und $d$, und so fort nach folgendem Schema:

$$
\begin{array}{r}
\left\{\begin{array}{l}
\mathbf{h} \\
\mathbf{d}^{\prime}
\end{array}\right. \\
\left\{\begin{array}{l}
\mathrm{g} \\
\mathrm{b}^{\prime \prime}
\end{array}\right. \\
\left\{\begin{array}{l}
\mathbf{d} \\
\mathbf{b} \\
\mathbf{b}^{\prime}
\end{array}\right. \\
\begin{array}{l}
\mathbf{b} \\
\mathbf{a}^{\prime}
\end{array} \\
\begin{array}{c}
\mathrm{c} \\
\mathbf{a}^{\prime \prime}
\end{array}\left\{\begin{array}{l}
\mathrm{e} \\
\mathbf{a}^{\prime \prime \prime},
\end{array}\right.
\end{array}
$$

so würde nichts entgegenstehen, dass die Reihe $\mathrm{a}^{\prime} \mathrm{a}^{\prime \prime} \mathrm{a}^{\prime \prime}$ sich dem Typus des Muttergewebes a ganz analog verhielte, die Derivate derselben $\mathbf{c}, \mathbf{c}^{\prime}$, e, f sich dem mehr mucinösen Charakter des Granulationsgewebes anschlössen, und die Reihe der Derivate $b, b^{\prime}, b^{\prime \prime}, d, d^{\prime}, g$, b allein in volle Suppuration geriethe. So stelit sich in der That in vielen Fallen das wirkliche Verhältniss heraus. Ja, es ist nicht schwer, Fälle zu finden, wo d' und h schon wieder fettig zerfallen, d. h. ihrerseits und in sich schmelzen. Dabei erbält sich in der Tiefe eine neugebildete, homologe Schicht, welche die Grundlage des nachherigen Regenerationsvorganges wird, gan $z$ in derselben Weise, wie diess in einem Geschwür stattindet, welches unter Eiterung durch Granulation beilt. Und somit muss ich allerdings daran festhalten, dass zwischen Suppuration (im Bindegewebe) und (suppurativer) Ulceration nur ein gradueller Unterschied besteht. 